EPJ Web of Conferences 33, 02009 (2012)

DOI: $10.1051 /$ epjconf/20123302009

(C) Owned by the authors, published by EDP Sciences, 2012

\title{
Determination of physical properties of fibrous thermal insulation
}

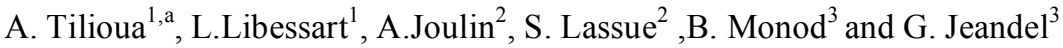 \\ ${ }^{1}$ LGCgE, HEI, F-59046 Lille, France \\ ${ }^{2}$ LGCgE, Faculté des Sciences Appliquées, Université d'Artois, 62400 Béthune, France \\ ${ }^{3}$ LEMTA, Nancy-Université, CNRS, Faculté des Sciences et Techniques, BP 239, 54506 \\ Vandoeuvre lès Nancy Cedex, France.
}

\begin{abstract}
The objective of this study is to characterize both experimentally and theoretically, conductive and radiative heat transfer within polyester batting. This material is derived from recycled bottles (PET) with fibres of constant diameters. Two other mineral and plant fibrous insulation materials, (glass wool and hemp wool) are also characterized for comparative purposes. To determine the overall thermophysical properties of the tested materials, heat flux measurement are carried out using a device developed in house. The radiative properties of the material are determined by an inverse method based on measurements of transmittance and reflectance using a FTIR spectrometer and by solving the equation of radiative heat transfer. These measures are compared to results of numerical simulations.
\end{abstract}

\section{Introduction}

Considering the environmental concerns, scientific research abounds in the area of the reduction of energy consumption and greenhouse-gas emissions in the building sector.

However, although the basic principles of insulation are well known, the study of the physical phenomena involved is usually over-simplified.

In addition the development of new innovative materials, the use of natural ingredients (plant fibers, etc...) and recycling offer a wide variety of opportunities and products with thermophysical properties difficult to predict and regulate. Optimizing the characteristics of such new materials requires good knowledge of basic physical phenomena, heat transfer and mass. As for heating, air captured within insulation, two coupled heat transfer modes (conduction and radiation) co-exist in a geometrically complex environment.

Not only are the intrinsic characteristics of fibers important, in addition an overall characterization of the environment is also required. The polyester fiber insulation is made up of cylindrical fibers distributed more or less randomly into the air.

This insulation is considered as a semi-transparent absorbing, emitting and scattering.

Bhattacharyya [1] estimated the existence of convection heat transfer in the calculation of the modified Rayleigh number Ra*, predicting the negligence of convection in fibrous material.

\footnotetext{
a e-mail : amine.tilioua@hei.fr
}

This is an Open Access article distributed under the terms of the Creative Commons Attribution License 2.0, which permits unrestricted use, distribution, and reproduction in any medium, provided the original work is properly cited. 
For insulation of porosity greater than or equal to $90 \%$ and at ambient temperature, the heat conduction of the gas with a contribution of $60 \%$ and $40-50 \%$ of thermal radiation are dominant heat transfer modes, conductive heat transfer is low.

The contribution of convection can often be overlooked. In order to optimize the energy efficiency of insulation and mainly fibrous insulation, it is important to control and monitor the radiative and conductive heat transfer. The problem of radiation coupled with other modes of heat transfer is encountered is many engineering applications.

For the thermal radiation of dispersed media, Sacadura and Baillis [2] have presented in review some trackers open by a new experimental methodology in the difficult area of accessment.

Most of current know is limited to room temperature property determination. In the other hand, Lee [3] developed a radiative model to evaluate the effect of fiber orientation on the radiative heat transfer through fibrous media between parallel planar diffuse boundaries.

Lee and Cunnington [4] have presented the improved theoretical model for the prediction of radiative properties and radiative heat transfer in high-porosity fiber thermal insulations and have demonstrated the validity of the model by comparing numerical predictions with a comprehensive database of experiments, most of them were obtained by other investigators before the present model was developed.

An inverse analysis to determine conductive and radiative properties of a fibrous medium is applied by Zhao et al [5] by changing the modification factor of extinction coefficient and an equivalent albedo of scattering were defined taking into account anisotropic scattering in fibrous insulation; they found that the calculated results corresponded well with the experimental data.

Let us mention that Cheheb et al. [6] have developed a theoretical and an experimental study allowing the measurement of the radiative and the conductive properties of semitransparent material by applying a crenel heating excitation and the Gauss-Newton method.

For a better understanding of the problem of identification of the radiative properties from measurements in two-way semi-transparent backgrounds with an acceptable number of parameters, Nicolau et al [7] proposed an inverse method consisting in determining spectral radiative properties of fibrous insulations by least-squares method for fiber randomly distributed in space and the phase function consisted of two Henyey and Greenstein functions coupled to an isotropic component. Milandri et al [8] applied this method to silica wool and showed that the inverse method can give similar results to the results obtained with the Mie theory, although in some cases, there are significant uncertainties due to the limits of the phase function used such as Henyey Greenstein functions associated with a Lorentz function. Finally, Kaemmerlen et al [9] applied the inverse method to determine radiative properties of the wood wool. Transient one-dimensional coupled radiative and conduction heat transfer was solved in a wood wool.

This paper is organized as follows. In the first section, we focus on laws governing the conduction and radiation by analyzing the equations of heat transfer and the apparent conductivity of the material according to the morphology of materials. In the second section, we describe the numerical model and the experimental measurement. Finally, experimental and numerical results will be presented. We conclude this work by the remarks and an outlook for further developments.

\section{Physical model}

The three modes of heat transfer within a material are radiation, conduction and convection. The fibrous media that we study here are made of two phases: a solid matrix containing fibres and a gas phase formed of air. The thermal transfer can take place by conduction through the solid matrix and the interstitial fluid, by natural convection via the movement of the interstitial fluid and by radiation. However, at ambient temperature, we can consider that the interstitial fluid is at rest and, therefore, that there is no convection movement. Hereafter, we will neglect convection. 
We will describe the radiation-conduction interaction inside a semi-transparent medium, two equations are necessary: the radiative transfer equation (RTE) and the energy conservation equation.

F. Asllanaj et al [10] used the Kirchhoff transformation associated with a P2 finite elements method to solve the non-transient Heat Conduction Equation and a direct method to solve the Radiative Transfer Equation, their numerical method is applied to any kind of fibrous media. In the absence of natural convective heat transfer, the conservation of energy for one-dimensional heat transfer through the insulation by conduction and radiation yields the partial differential equation:

$$
\rho C_{P} \frac{\partial T}{\partial t}(x, t)-\frac{\partial}{\partial x}\left(k(T(x, t)) \frac{\partial T}{\partial x}(x, t)\right)=S_{r}(x, t)
$$

This equation is coupled to the radiative transfer through the terms of radiative energy source

with the following initial and boundary conditions:

$$
S_{r}(x, t)=-\frac{\partial Q_{r}}{\partial x}(x, t)
$$

$$
\begin{aligned}
& T(x, 0)=T_{0} \\
& T(0, t)=T_{1} \\
& T(E, t)=T_{1}
\end{aligned}
$$

where T is temperature, $\rho$ is density, $C_{P}$ is specific heat, $k$ is thermal conductivity, $t$ is time, $x$ is the spatial coordinate through the insulation thickness and $\mathrm{E}$ is the insulation thickness. The total heat flux, given by the sum of the net radiation and conduction, is defined by

The radiant heat flux is given by

$$
Q_{t}(x, t)=Q_{r}(x, t)+Q_{c}(x, t)
$$

$$
Q_{r}(x, t)=2 \pi \int_{\lambda=0}^{\infty} \int_{\mu=-1}^{1} I_{\lambda}(x, \mu, t) \mu d \mu d \lambda
$$

and $I_{\lambda}(x, \mu, t)$ is determined from RTE as described below. The conduction heat flux is defined by

$$
Q_{c}(x, t)=-k(T(x, t)) \frac{d T(x, t)}{d x} \quad \forall 0<x<E
$$

$k(T)$ is the thermal conductivity depending on temperature. Langlais and Klarsfeld [11] have developed a semi-empirical relation for the thermal conductivity of fibrous insulation made up of the silica fibers and air:

$$
k(T)=2,572 \times 10^{-4} T^{0,81}+5,27 \times 10^{-5} \rho^{0,91}\left(1+1,3 \times 10^{-3} T\right)
$$

Where $\mathrm{T}$ is the temperature and $\rho$ is the density of the fibrous insulation. The tirst term is the thermal conductivity of the gas phase which corresponds to the conductivity of the air without movement and the second term is the thermal conductivity of the solid phase. This model (6) is an empirical relationship derived from measurements and regression.

In steady state, the energy becomes:

$$
\frac{d Q_{t}}{d x}(x)=0
$$

For a non-gray absorbing, emitting, and anisotropic scattering medium of thickness E, assuming heat transfer in the x-direction, with an axial symmetry as described in Modest [12] and Ozisik [13], is 


$$
\mu \frac{\partial L_{\lambda}(x, \mu)}{\partial x}=k_{\lambda} L_{\lambda}^{0}(x, \mu)-\beta_{\lambda} L_{\lambda}(T)+\frac{\sigma_{\lambda}}{2} \int_{-1}^{1} P_{\lambda}\left(\mu^{\prime} \rightarrow \mu\right) L_{\lambda}\left(x, \mu^{\prime}\right) \lambda \mu^{\prime}
$$

for $0<x<E, \mu \in[-1,1]\{0\}, \lambda>0, \mu$ is the cosine of the polar angle between the directions of the propagation and transfer. In this equation, the terms on the right hand side describe respectively internal emission, extinction phenomena and the intensity of the scattering in the $\mu$-direction. $L_{\lambda}^{0}(T)$ is the monochromatic intensity of the black body at temperature T, given by Planck's law as

$$
L_{\lambda}^{0}(T)=\frac{C_{1}}{\lambda^{5} \cdot\left[\exp \left(\frac{C_{2}}{\lambda \cdot T}\right)-1\right]}
$$

where $C_{1}$ and $C_{2}$ are two constants of the radiation

$$
C_{1}=1.19 \cdot 10^{-16} \mathrm{~W} / \mathrm{m}^{2} \text { and } C_{2}=1.4388 \cdot 10^{-2} \mathrm{~m} \cdot \mathrm{K}
$$

The monochromatic extinction coefficient is $\beta_{\lambda}=k_{\lambda}+\sigma_{\lambda} \cdot P\left(\mu^{\prime}, \mu\right)$ is the scattering phase function, wish is the fraction of radiative energy scattered into the outgoing direction, $\mu$, from the incoming direction, $\mu^{\prime}$

\section{Study on radiative heat transfer}

\subsection{Radiative properties}

The radiative properties provide information on the behavior of the fibrous medium such as a large optical thickness indicates a significant extinction of radiation in the environment.

For the determined radiative properties, we use the inverse method based on experimental measurement.

The radiative properties are identified from the three following physical quantities: directional hemispherical reflectance (DHR), directional hemispherical transmittance (DHT) and direct transmittance (DT).

The radiative properties of the medium are then characterized for each wavelength by optical thickness (calculated for $\mathrm{x}=\mathrm{E}$ in equation (18)), the albedo and the phase function. A numerical quadrature similar to the one used by Kaemmerlen et al [9] is employed.

The code consists in reducing the wavelength and calculating the theoretical quantities (DHR, DHT and DT) by solving the RTE (11) since they are all related to the spectral radiation intensity $L_{\lambda}$ Kaemmerlen et al [9].

$$
\begin{aligned}
& D H R_{\lambda}=-2 \pi \frac{\int_{-1}^{0} L_{\lambda}(0, \mu) \mu d \mu}{L_{0} d \Omega_{0}} \\
& D H T_{\lambda}=2 \pi \frac{\int_{0}^{1} L_{\lambda}(E, \mu) \mu d \mu}{L_{0} d \Omega_{0}}
\end{aligned}
$$




$$
D T_{\lambda}=\frac{L_{\lambda}\left(E, \mu_{1}\right)}{L_{0}}
$$

where $L_{0}$ is the incident radiation and intensity and $\mu_{1}$ is the first direction.

The principle of the inverse method consists in determining the radiative properties of a medium by minimizing the average standard deviation between theoretical and experimental DHR, DHT and DT. The function to be minimized is then written as

$$
F=\left[\frac{D H R_{\lambda, t h}-D H R_{\lambda, \exp }}{D H R_{\lambda, \exp }}\right]^{2}+\left[\frac{D H T_{\lambda, t h}-D H T_{\lambda, \exp }}{D H T_{\lambda, \exp }}\right]^{2}+\left[\frac{D T_{\lambda, t h}-D T_{\lambda, \exp }}{D T_{\lambda, \exp }}\right]^{2}
$$

Rosseland mean extinction coefficient: Experimental measurement on spectral transmission with FTIR are performed on three types of fibrous materials used for insulation. From the measurements, radiative heat conductivity is determined by calculating extinction coefficient using Beer's Law as (Siegel [13])

$$
T_{\lambda}=\exp \left(-\int_{0}^{E} \beta_{\lambda} d x\right)
$$

where $T_{\lambda}$ is the spectral transmittance and $\mathrm{E}$ is the thickness of the sample. For a homogeneous sample, $\beta_{\lambda}$ is independent of the sample thickness, then Eq. (17) can be reduced to $\beta(\mathrm{m}-1)$

$$
\beta_{\lambda}=-\frac{\ln \left(T_{\lambda}\right)}{E}
$$

The radiative conductivity $k_{r}$

$$
k_{r}=\frac{16 \sigma T_{m}^{3}}{3 \beta_{R}}
$$

where $T_{m}$ the mean temperature in the medium is, $\sigma$ is the Stefan-Boltzman constant and $\beta_{R}$ is the Rosseland average extinction coefficient given by:

$$
\frac{1}{\beta_{R}}=\int_{\lambda=0}^{\infty} \frac{1}{\beta_{\lambda}^{*}} \frac{d L_{\lambda}^{\circ}\left(T_{m}\right)}{d L^{\circ}\left(T_{m}\right)} d \lambda \cong \int_{b}^{a} \frac{1}{\beta_{\lambda}^{*}} \frac{d L_{\lambda}^{\circ}\left(T_{m}\right)}{d L^{\circ}\left(T_{m}\right)} d \lambda
$$

where $\beta_{\lambda}^{*}$ is the weight extinction coefficient, $L^{\circ}\left(T_{m}\right)$ is the blackbody intensity and $L_{\lambda}^{\circ}\left(T_{m}\right)$ is the spectral blackbody intensity at temperature $T_{m}$.

To take into account anisotropic scattering by insulation, a weighted spectral extinction coefficient $\beta_{\lambda}^{*}$ substitutes the real spectral extinction coefficient. The $\beta_{\lambda}^{*}$ is defined

$\beta_{\lambda}^{*}=k_{\lambda}+\sigma_{\lambda}^{*}$ and $\sigma_{\lambda}^{*}=\sigma_{\lambda}\left(1-g_{\lambda}\right)$

The optical thickness and weighted can also be defined:

$\tau_{\lambda}^{*}=\beta_{\lambda}^{*} z$ and $\omega_{\lambda}^{*}=\frac{\sigma_{\lambda}^{*}}{\beta_{\lambda}^{*}}$ 


\section{Results and discussion}

The experimental data are obtained for two thousand wavelengths. Only 50 values are kept in order to represent the global evolution of the experimental transmittance and reflectance.

The radiative properties of the medium polyester fiber are then characterized for each wavelength by the optical thickness $\tau$ (calculated for $\mathrm{z}=\mathrm{E}$ in equation (22)), the albedo $\omega$ and the phase function. Figue 1 shows the radiative properties identified with the inverse method. The extinction coefficient identified with a value close to the value given by the Beer-Lambert applied for each wavelength (Figure 2), the largest differences are observed between 16 and 20 microns,

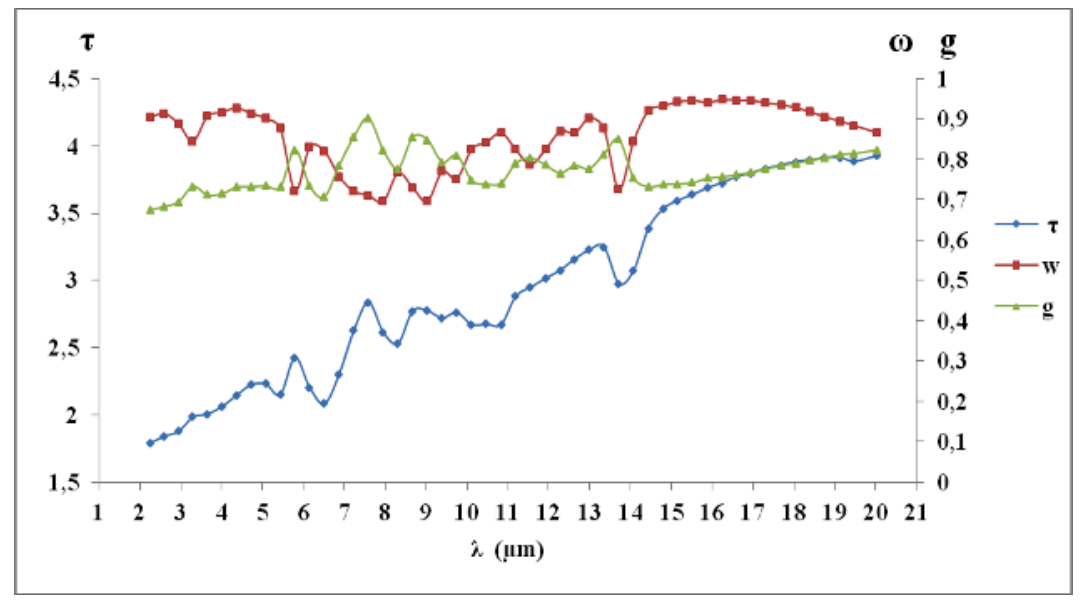

Fig. 1. Radiative properties of polyester fiber

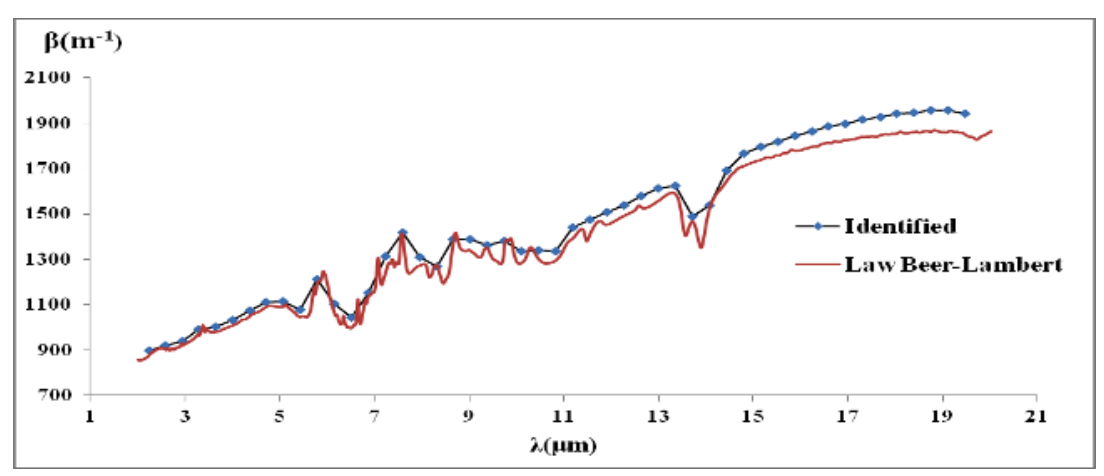

Fig. 2. Comparison of extinction coefficient identified and in relation to the Beer-

\section{Lambert's Polyester fiber}

The Rosseland equation is valid when the medium absorbs and scatters isotropically. The weighted radiative properties identified are shown in Figure 3, The absorption bands are between 3 and $4 \mu \mathrm{m}$ and between 15 and $17 \mu \mathrm{m}$.

The radiative conductivity of the polyester fiber was estimated from (20) on the wavelength range $\lambda=[2,20] \mu \mathrm{m}, k_{r}=0.0024 \mathrm{Wm}^{-1} \mathrm{~K}^{-1}$. The radiative conductivity then represents around $7 \%$ of the total thermal at $295 \mathrm{~K}$. 


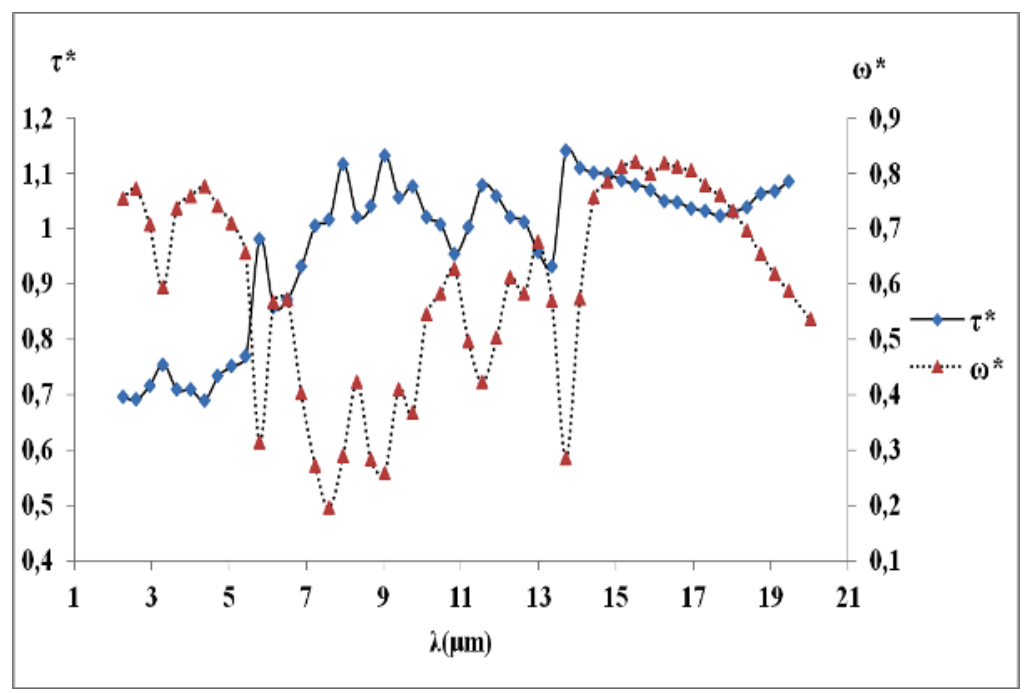

Fig. 3. Weighted radiative properties of polyester fiber

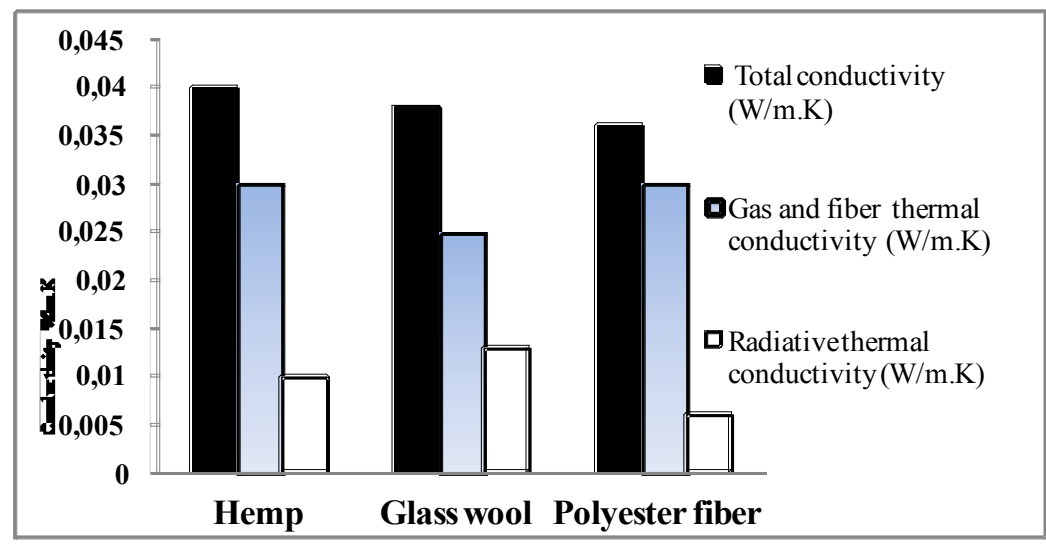

Fig. 4. Different thermal conductivities at the ambient temperature

We have determined the radiative properties for two materials, glass wool and hemp, with inverse method based on experimental measurements of reflection and transmission. The radiative conductivity is determined with Rosseland approximation. Figure 4. shows the different conductivities of three materials with different density of hemp, glass wool and fiber polyester. The total conductivity is known with accuracy by measurements thanks to the fluxmeter device taking into account the uncertainty of the measured total thermal conductivity. We note that the radiative conductivity has little influence on the total conductivity for the three materials. The radiative conductivity varies from product to product because of bulk density variation and also because of the variation of the insulation properties lumped in the specific scattering parameter. 


\section{Conclusion}

This work reports on experimental and numerical studies of thermal physical properties of fibrous insulation. The inverse method was successfully applied to determine the radiative properties of three fibrous materials (polyester fiber, hemp and glass wool) from FTIR measurements. The radiative conductivity was determined thanks to the Rosseland approximation. It is a low relative total conductivity for three materials fibrous, the radiative conductivity varies from material to material. A numerical model was used to simulate the steady-state and transient coupled heat transfer insulation. We plan in a future work to determine the radiative properties of the three materials based on a microscopic approach by Mie theory to compare it with the inverse method.

\section{References}

1. Bhattacharyya, R.K., Heat-transfer model for fibrous insulations, in:D.L. McElroy, R.P. Tye (Eds.), Thermal Insulation Performance, American Society for Testing and Materials, pp. 272$286(1980)$

2. Sacadura, J.F.,Baillis D. , Experimental characterization of thermal radiation properties of dispersed media Int. J. Therm. Sci. 41 699-707 (2002)

3. Lee, S. C. , Effect of fiber orientation on thermal radiation in fibrous media, J. Heat Mass Transfer Vol. 32, No. 2, pp. 311-319, (1989).

4. Lee, S.C. Cunnington, G.R. , Conduction and radiation heat transfer in high-porosity fiber thermal insulation, J Thermophys. Heat Transfer 14 (2) 121-136 (2000)

5. Zhao, S.Y., Zhang, B.M. and Du S.Y., An inverse analysis to conductive and radiative properties of a fibrous medium Journal of Quantitative Spectroscopy and Radiative Transfer, Volume 110, Issue 13, September, Pages 1111-1123 (2009)

6. Cheheb, Z., Albouchi, F. and Ben Nasrallah S., Measurement of thermal and conductive properties of semitransparent materials using a photothermal crenel method Journal of Quantitative Spectroscopy\& Radiative Transfer 109 620-635 (2008).

7. Nicolau VP, Raynaud M. and Sacadura JF., Spectral radiative properties identification of fiber insulating materials. International Journal of Heat and Mass Transfer ; 37:311-24(1994)

8. Milandri, A., Asllanaj, F. and G. Jeandel, Determination of radiative properties of fibrous media by an inverse method-comparison with the Mie theory, J. Quant. Spectrosc. Radiat. Transfer 74 637-653(2002)

9. Kaemmerlen, A., Asllanaj F., Sallée, H., Baillis, D. and Jeandel, G., Transient modeling of combined conduction and radiation in wood fibre insulation and comparaison with experimental data International Journal of Thermal Sciences 49 2169-2176 (2010).

10. Asllanaj, F., Jeandel, G., Roche, J.R. and Lacroix, D, Transient combined radiation and conduction heat transfer in fibrous media with temperature and flux boundary condition International Journal of Thermal Sciences 43 939-950 (2004).

11. C. Langlais and S. Klarsfeld,, Journée d'étude du groupement universitaire de thermique 6:19 (1985).

12. Modest MF. Radiative heat transfer, New York: McGraw-Hill, p. 61-4 (1993)

13. Seigel R, Howell, JR. Thermal radiation heat transfert, $3^{\text {rd }}$ ed. New York: Taylor \& Francis, p. 108-12 (1992) 